\section{Christmas disease in a Hovawart family resembling human hemophilia $B$ Leyden is caused by a single nucleotide deletion in a highly conserved transcription factor binding site of the F9 gene promoter}

Bertram Brenig ${ }^{1 *}$ Lilith Steingräber ${ }^{1 *}$ Shuwen Shan ${ }^{1}$ Fangzheng Xu, ${ }^{1}$ Marc Hirschfeld, ${ }^{1,2}$ Reiner Andag, ${ }^{3}$ Mirjam Spengeler, ${ }^{4}$ Elisabeth Dietschi, ${ }^{4}$ Reinhard Mischke ${ }^{5}$ and Tosso Leeb ${ }^{4}$

${ }^{1}$ University of Göttingen, Institute of Veterinary Medicine, Göttingen, Germany; ${ }^{2}$ Department of Obstetrics and Gynecology, Freiburg University Medical Center, Freiburg, Germany; ${ }^{3}$ University Medical Center Göttingen, Institute for Clinical Chemistry, Göttingen, Germany; ${ }^{4}$ Institute of Genetics, University of Bern, Bern, Switzerland and ${ }^{5}$ Small Animal Clinic, University of Veterinary Medicine Hannover Foundation, Hannover, Germany

${ }^{*} B B$ and LS contributed equally to this work.

\section{ABSTRACT}

$\mathrm{H}$ emophilia B is a classical monogenic, X-chromosomal, recessively transmitted bleeding disorder caused by genetic variants within the coagulation factor IX gene (F9). Although hemophilia B has been described in dogs, it has not yet been reported in the Hovawart breed. Here we describe the identification of a Hovawart family transmitting typical signs of an X-linked bleeding disorder. Five males were reported to suffer from recurrent hemorrhagic episodes. A blood sample from one of these males with only $2 \%$ of the normal concentration of plasma factor IX together with samples from seven relatives were provided. Next-generation sequencing of the mother and grandmother revealed a single nucleotide deletion in the F9 promoter. Genotyping of the deletion in 1,298 dog specimens including 720 Hovawarts revealed that the mutant allele was only present in the aforementioned Hovawart family. The deletion is located 73 bp upstream of the F9 start codon in the conserved overlapping DNA binding sites of hepatocyte nuclear factor $4 \alpha(\mathrm{HNF}-4 \alpha)$ and androgen receptor (AR). The deletion only abolished binding of HNF-4 $\alpha$, while AR binding was unaffected as demonstrated by electrophoretic mobility shift assay using human HNF- $4 \alpha$ and AR with double-stranded DNA probes encompassing the mutant promoter region. Luciferase reporter assays using wildtype and mutated promoter fragment constructs transfected into Hep G2 cells showed a significant reduction in expression from the mutant promoter. The data provide evidence that the deletion in the Hovawart family caused a rare type of hemophilia B resembling human hemophilia B Leyden.

\section{Introduction}

Hemophilia B (Christmas disease) is a recessive, X-linked bleeding disorder caused by genetic variants within the clotting factor IX gene (F9) resulting in the absence or insufficient levels of factor IX (FIX) in the blood. ${ }^{1}$ In humans hemophilia $\mathrm{B}$ is also known as the "royal disease" as it was transmitted into several European royal dynasties by Queen Victoria., ${ }^{2,3}$ As of present, 1,113 unique F9 variants have been described in humans. ${ }^{4}$ The majority of the pathogenic variants are located within exons $(\mathrm{n}=923)$ and intronic regions $(\mathrm{n}=137)$ of $F 9$. Only 33 variants $(2.96 \%)$ have been described in the 5'-UTR $(n=28)$ and 3'-UTR $(n=5)$ accounting for $2.52 \%$ and $0.45 \%$ of human pathogenic hemophilia $B$ variants, respectively. ${ }^{4}$

Although the first reports about canine hemophilia B date back to the early 1960s
Ferrata Storti Foundation

Haematologica 2019

Volume 104(11):2307-2313

\section{Correspondence:}

BERTRAM BRENIG

bbrenig@gwdg.de

Received: December 26, 2018

Accepted: March 6, 2019.

Pre-published: March 7, 2019.

doi:10.3324/haematol.2018.215426

Check the online version for the most updated information on this article, online supplements, and information on authorship \& disclosures: www.haematologica.org/content/104/11/2307

\section{(C)2019 Ferrata Storti Foundation}

Material published in Haematologica is covered by copyright All rights are reserved to the Ferrata Storti Foundation. Use of published material is allowed under the following terms and conditions:

https://creativecommons.org/licenses/by-nc/4.0/legalcode. Copies of published material are allowed for personal or internal use. Sharing published material for non-commercial purposes is subject to the following conditions:

https://creativecommons. org//icenses/by-nc/4.0/legalcode, sect. 3. Reproducing and sharing published material for commercial purposes is not allowed without permission in writing from the publisher. 
and this was the first disorder in dogs characterized on the DNA level, data on hemophilia B cases in dogs remain rather scarce compared to data from humans. ${ }^{5.8}$ For instance, in the Cairn Terrier colony of the Francis Owen Blood Research Laboratory (University of North Carolina, Chapel Hill, NC, USA) a G>A transition (NC_006621.3:g.109,532,018G>A) in exon 8 causing an amino acid exchange (NP_001003323.1:p.Gly418Glu) that resulted in a complete lack of circulating FIX was detected in affected dogs. ${ }^{9}$ Due to a complete deletion of $F 9$ in a Labrador Retriever, FIX inhibitors were produced after transfusion of canine blood products. ${ }^{10}$ In a study of Pit Bull Terrier mixed breed dogs and Airedale Terrier dogs a large deletion of the entire 5' region of $F 9$ extending to exon 6 was found in the former and a $5 \mathrm{~kb}$ insertion disrupting exon 8 was described in the latter. ${ }^{11}$ As in the Labrador Retriever with hemophilia B, FIX inhibitors were produced in both breeds. A mild form of hemophilia B in German Wirehaired Pointers was caused by a $1.5 \mathrm{~kb}$ Line1 insertion in intron 5 of $F 9$ at position NC_006621.3:g.109,521,130. ${ }^{12}$ Until today, hemophilia B has been described in four mixed-breed dogs and nine dog breeds, i.e. German Shepherd, Lhasa Apso, Labrador Retriever, Rhodesian Ridgeback, Airedale Terrier, Cairn Terrier, Maltese, Mongrel and German Wirehaired Pointer. $^{9-17}$

In the canine cases analyzed so far on the DNA level, mutations have been observed only in exons and introns of F9, whereas alterations of the F9 promoter have not yet been reported. In humans promoter variants have been detected and result in the so-called hemophilia B Leyden characterized by low levels of FIX until puberty, whereas after puberty FIX concentrations rise to almost normal levels. ${ }^{18-20}$ Since its first description, the genetic background of human hemophilia B Leyden was elucidated by various studies identifying variants in different transcription factor binding sites in the Fq promoter including the androgen-responsive element (ARE), hepatocyte nuclear factor $4 \alpha$ (HNF4 $\alpha$ ), one cut homeobox (ONECUT1/2) and CCAAT/enhancing-binding protein $\alpha$ $(\mathrm{C} / \mathrm{EBP} \alpha)$ binding sites. ${ }^{21,22} \mathrm{HNF} 4 \alpha$ is a liver-enriched member of the nuclear receptor superfamily of liganddependent transcription factors and has been associated with several disorders, including diabetes, atherosclerosis, hepatitis, cancer, and hemophilia. ${ }^{23}$ Promoter analyses have identified at least 140 genes with HNF4 $\alpha$ binding sites. A recent, more detailed analysis using protein binding microarrays identified an additional 1,400 potential binding sites ${ }^{24,25}$ Hence, HNF $4 \alpha$ plays an important role in the regulation of numerous genes especially in the maintenance of many liver-specific functions. Liver-specific HNF4 $\alpha$-null mice have been used to study the involvement of hepatic HNF4 $\alpha$ in blood coagulation. In the murine model it was shown that expression of factors $\mathrm{V}$, XI, XII, and XIIIB depends directly on hepatic HNF4 $\alpha$ and FIX expression was decreased with significantly prolonged activated partial thromboplastin time (aPTT) ${ }^{26}$ Ten of the so far identified 28 5'-UTR variants (35.7\%) are located within the overlapping binding sites of the androgen receptor (AR) and HNF4 $\alpha$ in the human F9 promoter. ${ }^{4,21}$ Four variants at positions $-21,-20$ and - -19 only affect HNF $4 \alpha$ binding and all of them have been shown to cause hemophilia B Leyden. . $^{1977-30}$ The remaining six variants at positions $-26,-24$ and -23 , located in the overlapping region, cause the so-called hemophilia B
Brandenburg. ${ }^{31,32}$ Unlike the classical hemophilia B Leyden, FIX levels in patients with these variants cannot be restored by testosterone-driven AR activity and remain low after puberty with no clinical recovery. ${ }^{21,32}$

\section{Methods}

\section{Animals and genomic DNA isolation}

Canine blood and/or hair samples were collected by local veterinarians. The collection of samples was approved by the Lower Saxony State Office for Consumer Protection and Food Safety (33.19-42502-05-15A506) according to $₫ 8 a$ Abs. 1 Nr. 2 of the German Animal Protection Law (TierSchG). Blood collected into EDTA and/or hair samples were provided by different Hovawart and dog breeders with written consent from the dogs' owners. DNA was extracted from 30-50 hair roots using the OIAamp DNA Mini Kit (Qiagen, Hilden, Germany) according to the manufacturer's instructions. ${ }^{33}$ A salting out procedure was used to obtain DNA from the EDTA blood samples. ${ }^{34}$ Additional DNA samples deposited with the Institute of Veterinary Medicine were used as controls. All samples were pseudonymized using internal identities.

\section{Next-generation sequencing and genotyping}

DNA from animals \#4 and \#6 was used for next-generation sequencing on an Illumina HiSeq2500. The quality of the fastqfiles was analyzed using FastQC 0.11.7..$^{35}$ Total reads of 1,029,601,630 (\#4; sequencing depth 51x) and 1,000,503,256 (\#6; sequencing depth 50x) were obtained and mapped to the reference canine F9 gene (NC_006621.3, region 109,501,341 to 109,533,798; CanFam3.1) using DNASTAR Lasergene Genomics Suite SeqMan NGen 15.2 .0 (130). . $^{36-40}$

Targeted genotyping of the promoter deletion was done by polymerase chain reaction (PCR) amplification with primers cfa_F9_Ex1_F (5'-CCACTGAGGGAGATGGACAC-3') and cfa_F9_Ex1_R (5'-CCCACATGCTGACGACTAGA-3') resulting in a fragment of $328 \mathrm{bp}$ (wildtype) or $327 \mathrm{bp}$ (deletion) spanning the variant position. The resulting PCR products were either directly sequenced on an ABI 3730 Genetic Analyzer (Thermo Fisher Scientific, Basel, Switzerland) or genotypes were determined by restriction fragment length polymorphism analysis after cleavage with $R s a I$. The wildtype allele generated two fragments of $52 \mathrm{bp}$ and $276 \mathrm{bp}$ while the allele with the deletion remained uncut.

\section{Electrophoretic mobility shift assay}

For the electrophoretic mobility shift assay, biotin-labeled, double-stranded wildtype (cfa_F9n_wt_Biotin: 5'-CAGAAGTAAATACAGCTCAACTTGTACTTTGGAACAACTGGTCAACC-3') and mutated (cfa_F9n_mut_Biotin: 5'-CCAGAAGTAAATACAGCTCAACTTGTATTTGGAACAACTGGTCAACC-3') oligonucleotides were synthesized (Integrated DNA Technologies IDT, Leuven, Belgium) harboring the overlapping HNF4 $\alpha$ and AR binding sites (underlined). The position of the deleted Cnucleotide is indicated in bold and italics. Recombinant human $\mathrm{HNF} 4 \alpha$ and human AR overexpression lysate were purchased from Origene Technologies Inc. (Rockville, MD, USA).

DNA was detected using the Chemiluminescent Nucleic Acid Detection Module Kit (Thermo Scientific, USA) with minor modifications, i.e. membranes were incubated for $1 \mathrm{~min}$ in the substrate working solution.

\section{Luciferase assay}

pGL3 Luciferase Reporter Vectors (pGL3-Basic, pGL3-Control) 
were used for the luciferase assay (Promega, Mannheim, Germany). The wildtype F9 promoter fragment (971 bp wildtype) was generated by PCR using primers cfa9_HindIIIF_neu (5'CGTAGACTTA GCACT GTTCAAAGCTTCACACA CACAGTTCTTAAAT-3') and cfa9_HindIII_R_neu (5'-ATGGCTAGCAACCGTCTAAGAAGCTTAATTGTGCAAGGAGCAAGG-3'). The mutated F9 promoter fragment ( $970 \mathrm{bp})$ was generated by PCR using primers cfa9_HindIII_F (5'-ATCGTCAAGCTTCACACACACAGTTCTTAAAT-3')

and cfa9_HindIII_R (5'-CGTACGAAGCTTAATTGTGCAAGGAGCAAGG-3'). For cloning into the HindIII restriction site of pGL3, primers were designed with an unspecific random 5'-tag (italics) followed by a HindIII restriction site (underlined) (Online Supplementary Figure S1). DNA from female carrier \#6 served as a template for amplification. Low expression levels of C/EBP in Hep G2 cells were complemented by co-transfection of a C/EBP $\alpha$ expression vector. ${ }^{22}$ Data are presented as relative response ratios. ${ }^{41}$ A Mann-Whitney $U$ test was used to determine statistical significance. Values were considered statistically significant when $P<0.05$ (weakly significant), $P<0.01$ (medium-strength significance) and $P<0.001$ (strongly significant).

\section{Other methods}

Further details of the study methods are given in the Online Supplementary Appendix.

\section{Results}

Hemophilias are rare diseases in dogs and hence it was rather coincidental that a case in a Hovawart (\#3, Figure 1) was reported to us. With the reconstruction of the pedigree using the online dog breed database and pedigree data of individual dogs provided by the owners it was possible to trace the disease back to the female carrier \#39 (Figure 1, Online Supplementary Figure S2). ${ }^{42}$ In the studied family the hemophilia was transmitted to animals \#19, \#4 and \#6. Bitch \#19 had one litter with three hemophilic males (\#48, \#51, \#53). Bitches \#4 and \#6 had litters with one affected male each, \#60 and \#3, respectively. Although DNA samples from animals \#48, \#51, \#53 and \#60 were not available, blood parameters and medical reports about recurrent hemorrhagic episodes were provided (Online

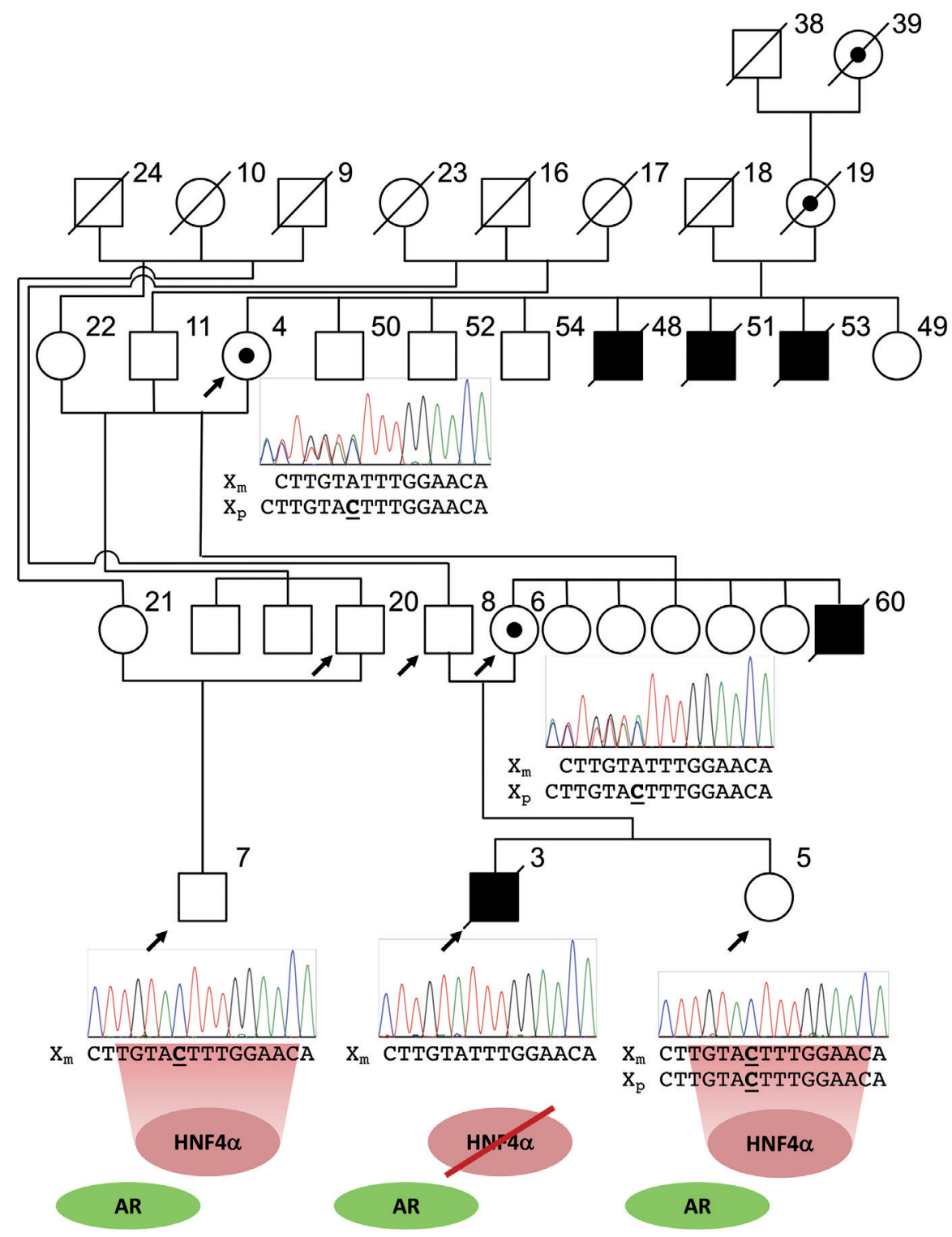

Figure 1. Pedigree section of the hemophilia B Leyden Hovawart family and DNA sequence comparison of the mutant hepatocyte nuclear factor $4 \alpha /$ androgen receptor binding site in the promoter of canine F9 in the hemophilic male (\#3) and relatives (\#4 grandmother, \#5 sister, \#6 mother, \#7 cousin). Pedigree symbols are according to the standardized human pedigree nomenclature. ${ }^{61}$ Individuals are pseudonymized using internal identities. DNA samples were available from individuals indicated with an arrow. DNA sequences of heterozygous bitches \#4 and \#6 (female carriers) show overlapping peaks with similar heights 5 ' of the deletion position. For males \#48, \#51, \#53 and \#60, signs of hemophilia (Online Supplementary Table S1) were reported and the dogs had to be euthanized after recurrent hemorrhages. $X_{m}$ : maternal $X$ chromosome; $X_{p}$ : paternal $X$ chromosome; HNF4 $\alpha$ : hepatocyte nuclear factor $4 \alpha$ binding site (consensus sequence: 5'-TGNACTTTG-3'); ${ }^{21,48}$ AR: 3'-part of the androgen receptor binding site (consensus sequence: 5'AGNACANNNTGTNCT-3'). ${ }^{21,48}$ 
Supplementary Table S1). These males had increased aPTT ratios of 2.93 (\#53) to 4.76 (\#51) indicative of defects in the intrinsic coagulation pathway and also reduced FIX concentrations in the blood as is normally the case in hemophilia B. The affected dog \#3 presented only $2 \%$ of the standard FIX concentration. The female carriers \#4 and \#6 showed aPTT ratios within the reference range. FIX concentrations, however, were slightly below the reference range (\#6). This was not surprising as it has been shown that minute reductions in FIX concentrations might not always be reflected in an aPTT increase because of the sensitivity of commercial reagents. ${ }^{43}$ The clinical signs together with the blood coagulation parameters and Xlinked transmission supported the diagnosis of hemophilia $\mathrm{B}$. The definite clinical diagnosis prompted us to search for the molecular cause initially on the DNA level. The canine Fq gene is located on chromosome X (CFAX) between positions 109,501,341 (transcription start site) and $109,533,798$ and has a length of 32,458 bp (NC_006621.3, CanFam3.1). The canine F9 gene, similarly to that of other mammals, has eight exons with an open reading frame of $1,356 \mathrm{bp}$ coding for 452 amino acids. ${ }^{44}$ DNA of female carriers \#4 and \#6 was subjected to whole genome sequencing and aligned to the canine reference Fo gene sequence. Surprisingly, only six sequence variants outside the coding regions of $F 9$ were identified (Table 1). Five variants were located in introns and were excluded as the cause of the hemophilia B in the Hovawarts because these variants were also detected in unaffected controls. The remaining variant (deletion) was located in the promoter of F9 73 bp upstream of the start codon (Online Supplementary Figures S1 and S3). As this deletion was located within a putative transcription factor binding site of HNF $4 \alpha$ and AR, which had been shown in humans to be important for F9 expression and mutated in hemophilia B Leyden and Brandenburg, ${ }^{31,32}$ this position was analyzed in more detail.

Figure 1 shows the segregation of the nucleotide deletion in the affected Hovawart family. The female carriers \#4 and \#6 were heterozygous, as evidenced by the overlap- ping peaks with similar heights $5^{\prime}$ of the deletion position. The affected male \#3 was hemizygous for the deleted allele whereas his sister \#5 and cousin \#7 were homozygous wildtype. Genotyping of 1,298 dogs (including 83 different breeds, 720 unrelated Hovawarts, and 12 Hovawart family members) demonstrated the occurrence of the deletion only among members of the affected Hovawart family (Table 2, Online Supplementary Table S2). To provide proof that the deletion represented the causative genetic variant and resulted in the low expression of F9, functional analyses using electrophoretic mobility shift and luciferase reporter assays were performed.

As shown in Figure 2, no binding of recombinant $\mathrm{HNF} 4 \alpha$ to the mutated promoter region was detected. On the other hand, the AR lysate clearly showed binding to both fragments and hence the deletion seems not to influence $\mathrm{AR}$ binding to the androgen-responsive element in the canine F9 promoter. To analyze the effect of the promoter variant on F9 expression, wildtype and mutated promoter fragment luciferase constructs were transfected into Hep G2 cells. As shown in Figure 3 the mutated promoter fragment resulted in a statistically highly significant $(P=2.2 \times 10$ $\left.{ }^{6}\right)$ reduction of the relative response ratio to approximately $34.6 \%$ of the wildtype promoter in the presence of C/EBP (+ C/EBP). C/EBP is clearly also an important transcription factor in the regulation of the canine $F 9$ promoter as shown when C/EBP was not co-transfected (- C/EBP). In the absence of C/EBP the relative response ratio of the wildtype promoter was $29.8 \%$. On the other hand there were no significant differences between the mutated promoter fragment (+/- C/EBP) and the wildtype promoter fragment (- C/EBP). As for variants of the HNF4 $\alpha$ site, disruption of the $\mathrm{C} / \mathrm{EBP}$ binding site has also been shown to be causative for hemophilia B in humans. ${ }^{45,46}$

\section{Discussion}

As in humans, hemophilia $\mathrm{A}$ and $\mathrm{B}$ are also rare diseases in dogs caused by sequence variants in the coagulation

Table 1. DNA sequence variants in the canine $F 9$ gene determined by next-generation sequencing of DNA of animals \#4 and \#6.

\begin{tabular}{lccl} 
Position & Ref/Alt & Gene region & HClN ${ }^{b}$ g. \\
X:109501492 & C/- & 5'-flanking region & NC_006621.3:g.109501492delC \\
X:109504229 & C/- & intron 1 & NC_006621.3:g.109504229delC \\
\hline X:109505462 & -/AG & intron 1 & NC_006621.3:109505462_109505463insAG \\
X:109507446 & -/A & intron 2 & NC_006621.3:109507446_109507446insA \\
\hline X:109510986 & G/A & intron 3 & NC_006621.3:g.109510986G $>$ A \\
X:109524055 & A/G & intron 6 & NC_006621.3:g.109524055A $>$ G
\end{tabular}

${ }^{\mathrm{a}}$ Ref/Alt: reference nucleotide/alternate nucleotide; ${ }^{\mathrm{b} H G V S}$ : Human Genome Variation Society (http://www.hgvs.org).

Table 2. F9 genotype frequencies

\begin{tabular}{|c|c|c|c|c|c|}
\hline \multirow[b]{2}{*}{ Cenotype } & \multicolumn{4}{|c|}{ Hovawart } & \multirow{2}{*}{$\begin{array}{c}\text { Other breeds } \\
\text { Controls }(n=567)\end{array}$} \\
\hline & $\mathrm{HB}^{\mathrm{a}}$ affected $(\mathrm{n}=1)$ & HB carrier $(n=2)$ & Control, related ( $n=12$ ) & $\begin{array}{l}\text { Control, unknown } \\
\text { relationship ( } n=720)\end{array}$ & \\
\hline $\mathrm{C} / \mathrm{C}$ & & & 12 & 720 & 567 \\
\hline C/- & & 2 & & & \\
\hline$-1-$ & 1 & & & & \\
\hline
\end{tabular}

${ }^{\mathrm{a} H B}$ : hemophilia B; ${ }^{\mathrm{b}} \mathrm{dog}$ breeds used as controls are listed in Online Supplementary Table S2. 


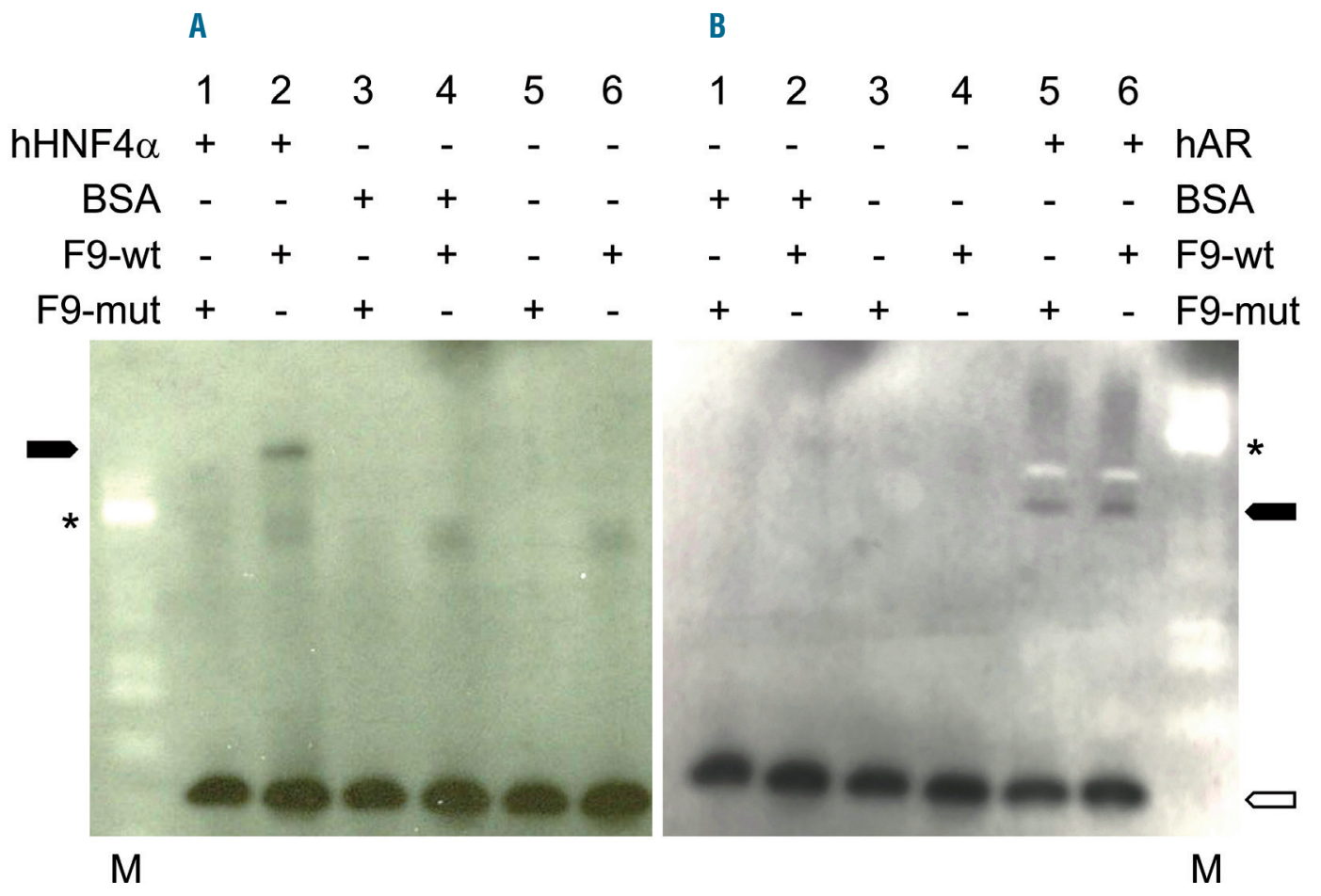

Figure 2. Analysis of hepatocyte nuclear factor $4 \alpha$ and androgen receptor binding of wildtype and mutated F9 promoter regions using an electrophoretic mobility shift assay. (A, B) Human hepatocyte nuclear factor $4 \alpha(\mathrm{HNF} 4 \alpha)$ and (B) androgen receptor (AR) were used to bind biotin-labeled wildtype and mutated $F 9$ promoter fragments (F9-wt, F9-mut). Specific shifted bands (solid arrowheads) are detected in lane 2 (A) for HNF4 $\alpha$ and lanes 5 and 6 (B) for AR. To test specificity, binding reactions were also performed using bovine serum albumin [BSA; lanes 3 and 4 (A), lanes 1 and 2 (B)]. In lanes 5 and 6 (A) and lanes 3 and 4 (B) no protein was added. Binding reactions were separated on $12 \%$ Tris-Glycine gels. X-ray films were cropped using GIMP 2.8.22. The 70 kDa protein marker band (PageRuler Prestained Protein Ladder, Fermentas) is indicated with an asterisk (lane M). The open arrowhead indicates unbound, free DNA.

factor VIII (F8) and IX (Fq) gene, respectively. ${ }^{47}$ Since the 1960s cases of canine hemophilia B have been reported and in 1989 the first description of the molecular cause in a Cairn Terrier population at the Francis Owen Blood Research Laboratory was published. $5,8,9,44$

Since then only six further types of variants, all of them affecting the coding region of the Fq gene, i.e. deletions, insertions, missense variants, have been described as causative for canine hemophilia B..$^{10-12,15,16}$ The identification of a causative promoter variant in the Hovawart dogs described here is therefore unique in two respects: it is the first regulatory variant described in dogs and secondly this variant resembles a specific subtype of hemophilia $B$, known as hemophilia B Leyden, in humans. ${ }^{21}$ Hitherto, 21 distinct variants in the human F9 promoter have been determined in families affected by hemophilia B Leyden. ${ }^{48}$ These variants cluster in the so-called Leyden-specific region (LSR) and interfere with the binding of different transcription factors, e.g. AR, HNF $4 \alpha$, ONECUT, and $\mathrm{C} / \mathrm{EBP} \alpha{ }^{49}$ The deletion identified in the Hovawart dogs was located $73 \mathrm{bp}$ upstream of the start codon of the canine F9 gene corresponding to position -23 in the third human promoter cluster harboring the overlapping binding sites of $\mathrm{AR}$ and $\mathrm{HNF} 4 \alpha{ }^{48}$ Similar to analyses in humans, it was possible to show by electrophoretic mobility shift assay that the deletion in the canine promoter abolished HNF $4 \alpha$ binding because it affects the highly conserved core sequence of the HNF $4 \alpha$ binding motif. ${ }^{24}$ On the other hand, binding of AR was not affected. This might be due to the fact that AR DNA-binding

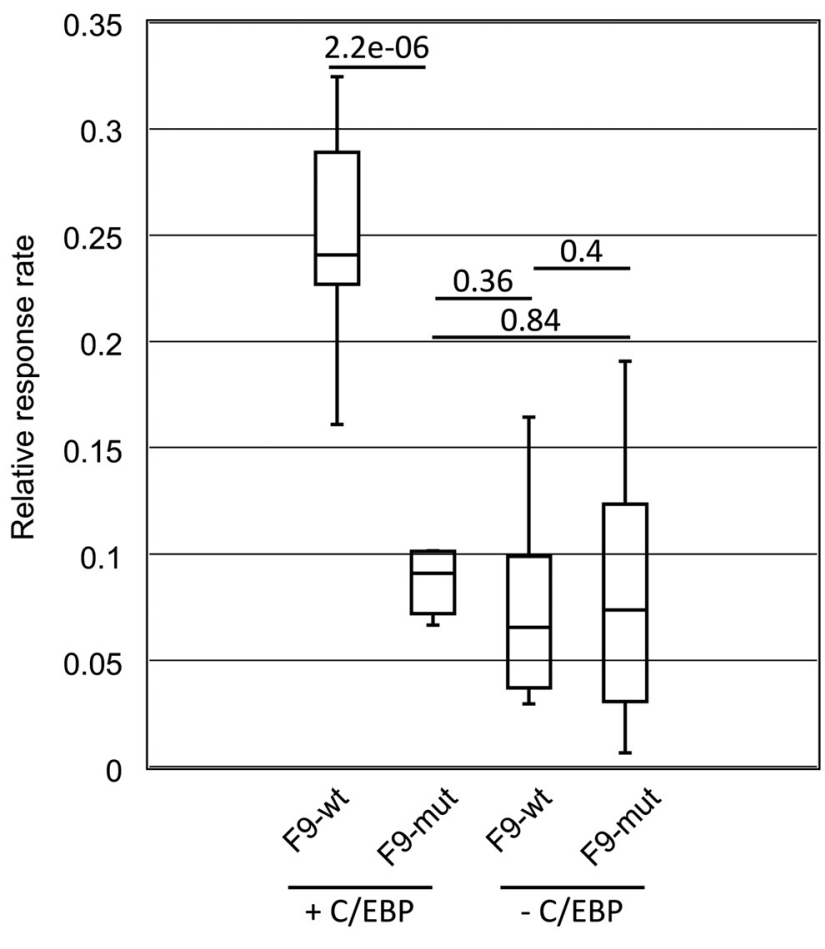

Figure 3. Dual-luciferase reporter analysis of F9 promoter activities in Hep G2 cells. Box and whisker plot showing the change of relative response ratios (RRR) between the wildtype (F9-wt) and mutant promoter (F9-mut) gene constructs. The lines in the boxes represent the medians. Whiskers indicate minimum and maximum RRR values. Values were normalized as described in the Online Supplementary Methods. P-values are indicated. 
sites display an exceptional amount of sequence variation..$^{50}$ Although the $\mathrm{C}$-deletion is located in the consensus TGTNCT-motif of class I AR-binding sites several alternative motives, e.g. TGTTTC in the stomatin-like protein 3 gene or TGTATC in the prostate-specific antigen gene enhancer III region, have been reported..$^{50-52}$ Therefore, it can be assumed that the affected males would have recovered from hemophilia during puberty. To what extent a sex- or possibly also age-dependent amelioration of the hemophilia B Leyden, as described in humans and also a mouse model, might be present in the affected Hovawarts remains to be determined. ${ }^{49,53}$ In this respect the Hovawart family could also be of interest as a model in the comparative analysis of age-dependent normalization of $\mathrm{Fg}$ expression in symptomatic carriers of hemophilia B Leyden in humans.

To analyze whether the deletion not only affected HNF $4 \alpha$ binding but also resulted in a downregulation of F9 expression, we performed luciferase assays. Although relative response ratios do not directly reflect $F 9$ mRNA or FIX levels in vivo, silencing of the promoter activity due to the deletion was obvious from these experiments. Similar findings have been made using HNF $4 \alpha$-null mice. ${ }^{26}$ In the murine model it was shown that expression of factors $V$, XI, XII, and XIIIB directly depends on hepatic HNF4 $\alpha$. Northern blot analysis also demonstrated that F9 expression was decreased with a significantly prolonged aPTT in the $\mathrm{HNF} 4 \alpha$-null mice. ${ }^{26}$ The finding that F9 expression was not completely dependent on HNF4 $\alpha$ is in agreement with the observation that control of Fq transcription in mice and humans is complex and depends on a plethora of factors. ${ }^{26}$ For instance, in earlier studies it was shown, using DNaseI footprinting, that there are further binding sites of HNF $4 \alpha$ and other factors, e.g. ARP1, COUP/Ear3, in the human F9 promoter influencing F9 expression. ${ }^{54}$ However, the classical HNF $4 \alpha$ binding site at position -26 to -19 only binds HNF4 $\alpha$. When analyzing the canine F9 promoter using transcription factor binding site prediction algorithms, further potential binding motifs for $\mathrm{HNF} 4 \alpha$ and other transcription factors were predicted (data not shown). ${ }^{55-57}$ As ectopic expression of F9 in vivo can be excluded or at least ignored according to recent RNAsequencing analyses, ${ }^{58}$ a remaining reduced hepatic activity of the mutated promoter is in agreement with the clinical findings of residual FIX activity in the affected males (Online Supplementary Table S1) and the results of the electrophoretic mobility shift assay showing binding of AR in androgen-dependent promoter activation.

In summary, we have identified and elucidated the causative genetic variant for hemophilia B Leyden in Hovawarts. This is the first report on a single nucleotide deletion within the binding sites of HNF $4 \alpha$ and AR in the F9 promoter causing hemophilia B Leyden in dogs. As the deletion only abolishes the binding of HNF $4 \alpha$, it can be assumed that male dogs will most likely recover during puberty, as reported in humans. ${ }^{30,59,60}$ However, to prevent any risk of a further propagation of the disorder, genotyping of females is recommended in further breeding.

\section{Acknowledgments}

The authors are grateful to S. Pach for expert technical assistance and L. Binder for support. The owners of Hovawarts who have provided blood samples are thanked for their generous help. A. Leutz and E. Kowenz-Leutz are thanked for providing the $C / E B P \alpha$ expression vector. S. Shan and F. Xu are supported by a fellowship from the Chinese Scholarship Council (CSC).

\section{References}

1. Dolan G, Benson G, Duffy A, et al. Haemophilia B: where are we now and what does the future hold? Blood Rev. 2018;32(1):52-60

2. Green P. The 'royal disease'. J Thromb Haemost. 2010;8(10):2214-2215.

3. Rogaev EI, Grigorenko AP, Faskhutdinova G, Kittler EL, Moliaka YK. Genotype analysis identifies the cause of the "royal disease". Science. 2009;326(5954):817.

4. Rallapalli PM, Kemball-Cook G, Tuddenham EG, Gomez K, Perkins SI. An interactive mutation database for human coagulation factor IX provides novel insights into the phenotypes and genetics of hemophilia B. J Thromb Haemost. 2013;11(7): 1329-1340

5. Mustard JF, Rowsell HC, Robinson GA, Hoeksema TD, Downie HG. Canine haemophilia B (Christmas disease). Br J Haematol. 1960;6:259-266.

6. Mustard JF, Basser W, Hedgardt G, Secord D, Rowsell HC, Downie HG. A comparison of the effect of serum and plasma transfusions on the clotting defect in canine haemophilia B. Br J Haematol. 1962;8:36-42.

7. Parks BJ, Brinkhous KM, Harris PF, Penick GD. Laboratory detection of female carriers of canine hemophilia. Thromb Diath Haemorrh. 1964;12:368-376.

8. Rowsell HC, Downie HG, Mustard JF, Leeson JE, Archibald JA. A disorder resem- bling hemophilia B (Christmas disease) in dogs. J Am Vet Med Assoc. 1960;137:247250 .

9. Evans JP, Brinkhous KM, Brayer GD, Reisner HM, High KA. Canine hemophilia B resulting from a point mutation with unusual consequences. Proc Natl Acad Sci U S A. 1989;86(24):10095-10099.

10. Brooks MB, Gu W, Ray K. Complete deletion of factor IX gene and inhibition of factor IX activity in a labrador retriever with hemophilia B. J Am Vet Med Assoc. 1997;211(11):1418-1421.

11. Gu W, Brooks M, Catalfamo J, Ray J, Ray K. Two distinct mutations cause severe hemophilia B in two unrelated canine pedigrees. Thromb Haemost. 1999;82(4):1270-1275.

12. Brooks MB, Gu W, Barnas JL, Ray J, Ray K. A Line 1 insertion in the factor IX gene segregates with mild hemophilia $B$ in dogs. Mamm Genome. 2003;14(11):788-795.

13. Feldman DG, Brooks MB, Dodds WJ. Hemophilia B (factor IX deficiency) in a family of German shepherd dogs. J Am Vet Med Assoc. 1995;206(12):1901-1905

14. Kooistra HS, Slappendel RJ. [A young male mongrel with hemophilia-B (Christmas disease)]. Tijdschr Diergeneeskd. 1991;116(6): 281-285.

15. Mauser AE, Whitlark J, Whitney KM, Lothrop CD Jr. A deletion mutation causes hemophilia B in Lhasa Apso dogs. Blood. 1996;88(9):3451-3455.

16. Mischke R, Kuhnlein P, Kehl A, et al. G244E in the canine factor IX gene leads to severe haemophilia B in Rhodesian Ridgebacks. Vet J. 2011;187(1):113-118.

17. Nakata M, Sakai M, Sakai T. Hemophilia B in a crossbred Maltese dog. J Vet Med Sci. 2006;68(11):1223-1224.

18. Reitsma PH, Mandalaki T, Kasper CK, Bertina RM, Briet E. Two novel point mutations correlate with an altered developmental expression of blood coagulation factor IX (hemophilia B Leyden phenotype). Blood. 1989;73(3):743-746.

19. Reitsma PH, Bertina RM, Ploos van Amstel JK, Riemens A, Briet E. The putative factor IX gene promoter in hemophilia B Leyden. Blood. 1988;72(3):1074-1076.

20. Veltkamp JJ, Meilof J, Remmelts HG, van der Vlerk D, Loeliger EA. Another genetic variant of haemophilia B: haemophilia B Leyden. Scand J Haematol. 1970;7(2):82-90.

21. Funnell AP, Crossley M. Hemophilia B Leyden and once mysterious cis-regulatory mutations. Trends Genet. 2014;30(1):18-23.

22. Picketts DJ, Lillicrap DP, Mueller CR. Synergy between transcription factors DBP and C/EBP compensates for a haemophilia $B$ Leyden factor IX mutation. Nat Genet. 1993;3(2):175-179.

23. Yuan X, Ta TC, Lin M, et al. Identification of an endogenous ligand bound to a native orphan nuclear receptor. PLoS One. 2009;4(5):e5609.

24. Bolotin E, Liao H, Ta TC, et al. Integrated approach for the identification of human hepatocyte nuclear factor 4alpha target genes using protein binding microarrays. 
Christmas disease in a Hovawart family

Hepatology. 2010;51(2):642-653.

25. Weltmeier F, Borlak J. A high resolution genome-wide scan of HNF4alpha recogniton sites infers a regulatory gene network in colon cancer. PLoS One. 2011;6(7):e21667.

26. Indue $\mathrm{Y}$, Peters $\mathrm{LL}$, Mim $\mathrm{SH}$, Indue J, Gonzalez FJ. Role of hepatocyte nuclear factor alpha in control of blood coagulation factor gene expression. J Mol Med (Bert). 2006;84(4):334-344.

27. Belvini D, Salviato R, Radossi P, et al. Molecular genotyping of the Italian cohort of patients with hemophilia B. Haematological. 2005;90(5):635-642.

28. Ghanem N, Costed B, Martin J, et al. Twenty-four novel hemophilia B mutations revealed by rapid scanning of the whole factor IX gene in a French population sample. Eur J Hum Genet. 1993;1(2):144-155.

29. Kettering RP, Lu JZ, Liao D, et al. Two novel factor IX promoter mutations: incremental progress towards 'saturation in vive mutagenesis' of a human promoter region. Hum Mol Genet. 1995;4(4):769-770.

30. Reijnen MJ, Peerlinck K, Maasdam D, Bertina RM, Reitsma PH. Hemophilia B Leyden: substitution of thymine for guanine at position -21 results in a disruption of a hepatocyte nuclear factor 4 binding site in the factor IX promoter. Blood. 1993;82 (1):151-158.

31. Heir JA, Kettering RP, Zapata RE, Ordonez SM, Rasper CK, Sommer SS. Haemophilia B Brandenberg-type promoter mutation. Haemophilia. 1999;5(1):73-75.

32. Crossly M, Ludwig M, Stowell KM, De Voc P, Ole K, Brownie GG. Recovery from hemophilia B Leyden: an androgen-responsine element in the factor IX promoter. Science. 1992;257(5068):377-379.

33. Pfeiffer I, Volkel I, Taubert H, Brenig B. Forensic DNA-typing of dog hair: DNAextraction and PCR amplification. Forensic Sci Int. 2004;141(2-3):149-151.

34. Miller SA, Dykes DD, Polesky HF. A simple salting out procedure for extracting DNA from human nucleated cells. Nucleic Acids Res. 1988;16(3):1215.

35. Brown J, Pirrung M, McCue LA. FOC Dashboard: integrates Fast QC results into a web-based, interactive, and extensible FASTQ quality control tool. Bioinformatics. 2017 Jun 9. [Epub ahead of print]

36. Lindblad-Toh K, Wade CM, Mikkelsen TS, et al. Genome sequence, comparative analysis and haplotype structure of the domestic dog. Nature. 2005;438(7069):803-819.

37. Burland TG. DNASTAR's Lasergene sequence analysis software. Methods $\mathrm{Mol}$ Biol. 2000;132:71-91.

38. Clewley JP. Macintosh sequence analysis software. DNAStar's LaserGene. Mol Biotechnol. 1995;3(3):221-224.

39. Jiang M, Li H, Chang Y, et al. Transitional basal cells at the squamous-columnar junetion generate Barrett's oesophagus. Nature. 2017;550(7677):529-533.

40. Lawson B, Robinson RA, Fernandez JR, et al. Spatio-temporal dynamics and aetiology of proliferative leg skin lesions in wild British finches. Sci Rep. 2018;8(1):14670.

41. Eggers C, Hook B, Lewis S, Strayer C, Landreman A. Designing a bioluminescent reporter assay: normalization. 2016 [cited 14.11.2018]; Available from: http://www.promega.de/resources/pubhub/designing-a-bioluminescent-reporterassay-normalization/

42. Dögel M. Working Dog. 2019 [cited 01.02.2019]; Available from: https://en.working-dog.com

43. Mischke R. [Comparison of factor VIII:C and factor IX sensitivity of different commercial APTT reagents for canine plasma]. Burl Munch Tierarztl Wochenschr. 1999;112(10-11):394-399.

44. Evans JP, Watzke HH, Ware JL, Stafford DW, High KA. Molecular cloning of a eDNA encoding canine factor IX. Blood. 1989;74 (1):207-212.

45. Bentley AK, Rues DJ, Rizza C, Brownlee GG. Defective propeptide processing of blood clotting factor IX caused by mutation of arginine to glutamine at position -4. Cell. 1986;45(3):343-348.

46. Crossly M, Brownie GG. Disruption of a $\mathrm{C} / \mathrm{EBP}$ binding site in the factor IX promoter is associated with haemophilia B. Nature. 1990;345(6274):444-446.

47. Mischke R. [Haemophilia A and B in dogs]. Tierarztl Prox Aus K Kleintiere Heimtiere. 2012;40(1):44-53; quiz 54.

48. Funnell AP, Wilson MD, Ballester B, et al. A CPG mutational hotspot in a ONECUT binding site accounts for the prevalent variant of hemophilia B Leyden. Am J Hum Genet. 2013;92(3):460-467.

49. Kurachi S, Hoo JS, Amer A, Chang K, Yoshizawa AC, Kurachi K. An age-related homeostasis mechanism is essential for spontaneous amelioration of hemophilia B Leyden. Proc Natl Aced Sci U S A. 2009;106(19):7921-7926.

50. Reid KJ, Wendy SC, Sarto J, Sorensen P, Nelson CC. Two classes of androgen receptor elements mediate cooperativity through allosteric interactions. J Biol Chem. 2001;276 (4):2943-2952.

51. Wilson S, Qi J, Filipp FV. Refinement of the androgen response element based on ChIPSeq in androgen-insensitive and androgenresponsive prostate cancer cell lines. Sci Rep. 2016;6:32611.

52. Schuur ER, Henderson GA, Kmetec LA, Miller JD, Lamparski HG, Henderson DR. Prostate-specific antigen expression is regulated by an upstream enhancer. J Biol Chem. 1996;271(12):7043-7051.

53. Hildyard C, Keeling D. Effect of age on factor IX levels in symptomatic carriers of haemophila B Leyden. Br J Haematol. 2015;169(3):448-449.

54. Naka H, Brownie GG. Transcriptional regulation of the human factor IX promoter by the orphan receptor superfamily factor, HNF4, ARP1 and COUP/Ear3. Br J Haematol. 1996;92(1):231-240.

55. Fang B, Mane-Padros D, Bolotin E, Jiang T, Sladek FM. Identification of a binding motif specific to HNF4 by comparative analysis of multiple nuclear receptors. Nucleic Acids Res. 2012;40(12):5343-5356,

56. Fore D, Reset $R$, Huerta $M$, et al. Identification of patterns in biological sequences at the ALGGEN server: PROMO and MALGEN. Nucleic Acids Res. 2003;31(13):3651-3653.

57. Messeguer X, Escudero R, Fore D, Nunez O, Martinez J, Alba MM. PROMO: detecton of known transcription regulatory lemints using species-tailored searches. Bioinformatics. 2002;18(2):333-334.

58. Fagerberg L, Hallstrom BM, Oksvold P, et al. Analysis of the human tissue-specific expression by genome-wide integration of transcriptomics and antibody-based proteomics. Mol Cell Proteomics. 2014;13(2): 397-406.

59. Reijnen MJ, Sladek FM, Bertina RM, Reitsma $\mathrm{PH}$. Disruption of a binding site for hepatocyte nuclear factor 4 results in hemophilia $B$ Leyden. Proc Natl Aced Sci U S A. 1992;89(14):6300-6303.

60. Morgan GE, Rowley G, Green PM, Chisholm M, Giannelli F, Brownlee GG. Further evidence for the importance of an androgen response element in the factor IX promoter. Br J Haematol. 1997;98(1):79-85.

61. Bennett RL, French KS, Resta RG, Doyle DL. Standardized human pedigree nomenclatore: update and assessment of the recommentations of the National Society of Genetic Counselors. J Genet Coons. 2008;17(5):424-433.

haematological | 2019; 104(11)

2313 